\title{
The Microwave Spectrum of 3,4-Dihydro-1,2-Pyran
}

\author{
Juan Carlos López and José L. Alonso \\ Departamento de Química-Física, Facultad de Ciencias Universidad de Valladolid, \\ Valladolid, Spain
}

Z. Naturforsch. 40 a, 913-919 (1985); received March 18, 1985

\begin{abstract}
The rotational transitions of 3,4-dihydro-1,2-pyran in the ground state and six vibrationally excited states have been assigned. The rotational constants for the ground state $(A=5198.1847(24)$, $B=4747.8716(24)$ and $C=2710.9161(24)$ have been derived by fitting $\mu_{a}, \mu_{b}$ and $\mu_{c}$-type transitions. The dipole moment was determined from Stark displacement measurements to be $1.400(8) \mathrm{D}$ with its principal axis components $\left|\mu_{a}\right|=1.240(2),\left|\mu_{b}\right|=0.588(10)$ and $\left|\mu_{c}\right|=$ $0.278(8) \mathrm{D}$. A model calculation to reproduce the ground state rotational constants indicates that the data are consistent with a twisted ring conformation. The average intensity ratio gives vibrational separations between the ground and excited states of the ring-bending and ring-twisting modes of $\sim 178$ and $\sim 277 \mathrm{~cm}^{-1}$ respectively.
\end{abstract}

\section{Introduction}

The conformational behaviour of six-membered ring molecules has been of interest for many years. NMR, FIR, Raman and Microwave Spectroscopy, as well as Electron Diffraction, have been used to obtain information on the molecular structure, conformation, potential surface, dipole moments, etc.

3,4-Dihydro-1,2-pyran is a member of the pseudofive-membered ring molecules in which one $\alpha$ methylene group of cyclohexene is replaced by an oxygen atom. Studies of the microwave spectra of cyclohexene [1, 2], 2,3-dihydro-p-dioxin [3] and 3,6dihydro-1,2-pyran [4] have shown that the theoretically proposed [5-9] half-chair or twisted conformation is the most stable one for cyclohexane derivatives.

FIR [10] and Raman [11] spectra of 3,4-dihydro1,2-pyran have been analyzed on the basis of two highly coupled low frequency vibrations corresponding to the out-of-plane modes ring-bending and ring-twisting (see Fig. 1) in a similar way as in the case of hindered pseudorotation in cyclopentanone [12]. The resultant two-dimensional potential surface leads to values of the barriers to ring planarity and interconversion of the stable twisted form of 6160 and $2340 \mathrm{~cm}^{-1}$, respectively, as well as a twist angle $\tau$ of $51.04^{\circ}$. It involves that the in-

Reprint requests to J. C. López, Departamento de QuímicaFísica. Facultad de Ciencias, Universidad de Valladolid, E-47005 Valladolid, Spain.
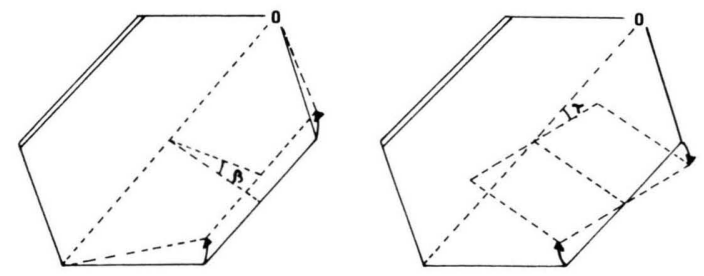

Fig. 1. The ring-bending $(\beta)$ and ring-twisting $(\tau)$ coordinates.

version process occurs through a metastable bent form, rather than through the planar configuration. According to the potential surface this bent form is $352 \mathrm{~cm}^{-1}$ less stable than the twisted one.

The barrier to interconversion is in good agreement with that obtained in a previous study of the temperature dependence of the NMR spectrum of 3,4-dihydro-1,2-pyran $\left(2308 \mathrm{~cm}^{-1}\right)$ [13].

We report a full analysis of the rotational spectrum of 3,4-dihydro-1,2-pyran in the ground and excited states of the ring vibrational modes. We establish conclusively from the ground state rotational constants a twisted equilibrium conformation for this molecule. The analysis of the excited states of the bending and twisting modes has provided interesting information about the skeletal ring modes. These results are also compared with those of cyclohexene [2], 2,3-dihydro-p-dioxin [3, 10,11] and 3,6-dihydro1,2-pyran.

A preliminary microwave study has been done by Sheridan [14], but no data have yet been reported. 


\section{Experimental}

The sample of 3,4-dihydro-1,2-pyran was supplied by Aldrich Chem. Co. and was used without further purification. The microwave spectrum was investigated in the $\mathrm{K}$-band region $(18.8$ to $26.5 \mathrm{GHz})$ by using a HP-8460 A MRR Spectrometer with a Stark modulation frequency of $33.3 \mathrm{kHz}$. The frequency accuracy was estimated to be better than $\pm 0.05 \mathrm{MHz}$. All observations were taken at room temperature with sample pressures in the range $10-50 \mu$. In the determination of the electric dipole moment, all ground-to-base voltage measurements were made using a HP-3469 B digital voltmeter. The arrangement of the radio-frequency microwave double resonance (RFMWDR) has been made in our laboratory and it is a modification of the system used by Wodarczyk and Wilson [15].

\section{Ground Vibrational State}

Preliminary rotational constants of the twisted and bent conformations of 3,4-dihydro-1,2-pyran were calculated by assuming structural parameters taken from 2,3-dihydro-p-dioxin [3] and cyclohexene [2]. These calculations indicated that 3,4-dihydro1,2 -pyran is a near asymmetric oblate $(\varkappa \simeq 0.6)$ with $a$ - and $b$-type transitions expected to be dominant. Experimentally, the rotational spectrum of the twisted conformer was only detected.

The dominant feature of the spectrum is an overlapping between $a$ - and $b$-type double Q-branch series. The branch heads, which are transitions of the $a$-type $J_{2, J-1} \rightarrow J_{0, J}, J_{1, J-1} \rightarrow J_{1, J}$ and $b$-type $J_{2, J-1} \rightarrow J_{1, J}, J_{1, J-1} \rightarrow J_{0, J}$, occur at intervals of 4.50 GHz. $\Delta K_{-1}=0 a$-type sub-branch and the corresponding $\quad K_{-1}+K_{+1}=J+1 \leftrightarrow K_{-1}+K_{+1}=J+1$ $b$-type sub-branch proceed continuously to lower frequencies with increasing $J$, but the $\Delta K_{-1}=2$ $a$-type sub-branch and the corresponding $K_{-1}+K_{+1}$ $=J \leftrightarrow K_{-1}+K_{+1}=J$ b-type sub-branch show decreasing frequency down to a value of $J$ from which the frequency starts to increase. These series were assigned by their characteristic Stark effect. A careful study of this series shows that the $a$ - and $b$-type lines become resolved with increasing $J$, the $a$-type series being the stronger one.

From these frequencies good values of $\frac{1}{2}(A-C)$ and $x$ were obtained. The R-branch assignment was obtained by identifying the $4_{0,4} \leftarrow 3_{0,3}, 4_{1,4} \rightarrow 3_{1,3}$ transitions, split by $5.7 \mathrm{MHz}$, and the $3_{0,3} \leftarrow 2_{0,2}$, $3_{1,3} \leftarrow 2_{1,2}$ transitions split, by $60.4 \mathrm{MHz}$, by means of the expected mirror image Stark pattern. These transitions were easily assigned by scanning at low Stark voltage. This assignment was also confirmed by RFMWDR. Further $a-, b$ - and $c$-type R-branch transitions were then assigned. The $c$-type lines are much weaker in intensity and although some of them were observed, it was possible to measure only two of them because of the high density of the spectrum.

The identification of many high- $J$ ( $J \leqq 35)$ of Rand Q-branch transitions has allowed the centrifugal distortion analysis of the spectrum. A Watson-type [16] treatment, using the III $l$ representation [17], has been used to take the centrifugal distortion effect into consideration. The effective rotational constants as well as the quartic distortion coefficients are collected in Table 1. (The list of the measured frequencies as well as their deviations from those calculated by using the constants of Tables 1 and 5 can be obtained from the authors on request.)

\section{Dipole Moment}

To determine the dipole moment, Stark effect measurements were made on ten components belonging to four transitions. These components are relatively free from interferences and were observed to have a strictly second order Stark effect. The Stark coefficients were calculated by the method of Golden and Wilson [18]. The electric field strength in the absorption cell was calibrated using the $M=1$ and $|M|=0$ components of the $2 \leftarrow 1$ transition of OCS with a dipole moment of $0.71521 \mathrm{D}$

Table 1. Rotational constants $(\mathrm{MHz})$, principal inertial moments $\left(\mathrm{amu} \mathrm{A} \mathrm{A}^{2}\right.$ ) and centrifugal distortion constants $(\mathrm{kHz})$ for the ground vibrational state of 3,4-dihydro1,2-pyran.

\begin{tabular}{lrll}
\hline$A$ & $5198.184(2)$ & $\Delta_{J}$ & $1.4(1)$ \\
$B$ & $4747.871(2)$ & $\Delta_{J K}$ & $-2.149(2)$ \\
$C$ & $2710.916(2)$ & $\Delta_{K}$ & $1.11(1)$ \\
$I_{a}$ & $97.2222(1)$ & $\delta_{J}$ & $-0.0835(2)$ \\
$I_{b}$ & $106.4433(1)$ & $\delta_{K}$ & $1.613(3)$ \\
$I_{c}$ & $186.4237(1)$ & $\sigma^{\mathrm{c}}$ & 0.020 \\
$\Delta_{0}=I_{c}-I_{a}-I_{b}$ & $-17.2418(2)$ & $N^{\mathrm{d}}$ & 156 \\
\hline
\end{tabular}

a Conversion factor $=505379.1 \mathrm{MHz}$ amu $\mathrm{A}^{2}$.

b Standard errors in parenthesis on the last digit.

Standard deviation of the fit.

d Number of measured transitions. 
Table 2. Correlation matrix for the ground state.

\begin{tabular}{lrrrrrrr}
\hline$A$ & 1.0000 & & & & & & \\
$B$ & 0.9729 & 1.0000 & & & & & \\
$C$ & 0.9669 & 0.9985 & 1.0000 & & & & \\
$\Delta_{J}$ & 0.9249 & 0.9286 & 0.9255 & 1.0000 & & & \\
$\Delta_{J K}$ & -0.0440 & 0.0374 & 0.0170 & 0.036 & 1.0000 & 1.0000 & \\
$\Delta_{K}$ & 0.1650 & -0.0353 & -0.0438 & 0.0368 & -0.6946 & 1.0000 & \\
$\delta_{J}$ & 0.0669 & 0.0225 & -0.070 & 0.0420 & 0.7717 & -0.1067 & -0.9566 \\
$\delta_{k}$ & -0.0434 & -0.0200 & 0.0095 & -0.0316 & -0.7861 & 0.1756 & 1.0000 \\
\hline
\end{tabular}

Table 3. Stark coefficients $\left(10^{5} \mathrm{MHz} \mathrm{cm}^{2} \mathrm{~V}^{-2}\right)$ and dipole moment components (D) for the ground state of 3,4-dihydro-1,2-pyran.

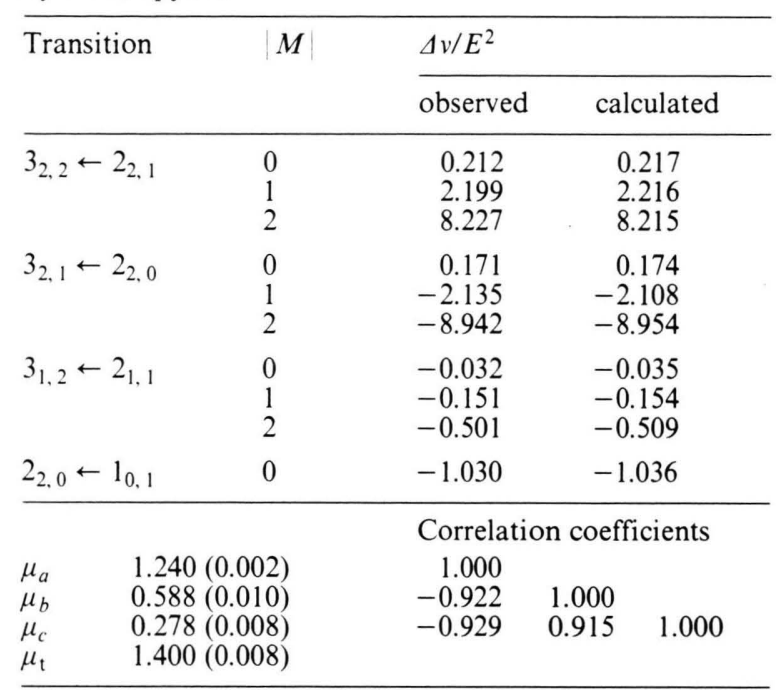

a Standard errors.

[19]. The squared dipole moment components $\mu_{a}^{2}, \mu_{b}^{2}$ and $\mu_{c}^{2}$ were obtained from a least squares fit of the observed second-order coefficients $\Delta v / E^{2}$. The transitions, Stark components, and coefficients are given in Table 3. The calculated total dipole moment is 1.400(8) D.

\section{Molecular Structure}

The non-planarity of the heavy atoms in the vibrational ground state is readily established from the quantity $\Delta_{0}=I_{c}-I_{a}-I_{b}$. If zero-point vibrational effects are neglected and a $\mathrm{C}-\mathrm{H}$ bond length of $1.096 \AA$ and a tetrahedral angle of $109^{\circ} 28^{\prime}$ are assumed for the methylene group parameters, $\Delta_{0}=$ $I_{c}-I_{a}-I_{b}$ can be calculated to be $-9.686 \mathrm{amu} \AA^{2}$. The observed value of -17.2418 amu $\AA^{2}$ must contain an appreciable contribution from the ring
Table 4. Plausible structural parameters ${ }^{a}$, observed, and predicted constants of 3,4-dihydro-1,2-pyran.

\begin{tabular}{llll}
\hline Bond & Length $(\AA)$ & Angle & Value $\left(^{\circ}\right)$ \\
\hline $\mathrm{O}_{1}-\mathrm{C}_{2}$ & $1.405^{\mathrm{b}}$ & $<\mathrm{O}_{1} \mathrm{C}_{2} \mathrm{C}_{3}$ & $123.4^{\mathrm{b}}$ \\
$\mathrm{O}_{1}-\mathrm{C}_{6}$ & $1.42^{\mathrm{b}}$ & $<\mathrm{C}_{2} \mathrm{C}_{3} \mathrm{C}_{4}$ & $123.3^{\mathrm{b}}$ \\
$\mathrm{C}_{2}-\mathrm{C}_{3}$ & $1.338^{\mathrm{b}}$ & $<\mathrm{C}_{3} \mathrm{C}_{4} \mathrm{C}_{5}$ & $110.3^{\mathrm{b}}$ \\
$\mathrm{C}_{3}-\mathrm{C}_{4}$ & $1.51^{\mathrm{b}}$ & $<\mathrm{C}_{6} \mathrm{O}_{1} \mathrm{C}_{2}$ & $111.6^{\mathrm{b}}$ \\
$\mathrm{C}_{4}-\mathrm{C}_{5}$ & $1.53^{\mathrm{b}}$ & $<\mathrm{HCH}$ & $109^{\mathrm{b}}$ \\
$\mathrm{C}_{5}-\mathrm{C}_{6}$ & $1.516^{\mathrm{c}}$ & $\theta$ & $17.0^{\mathrm{c}}$ \\
$\mathrm{C}-\mathrm{H}$ & $1.09^{\mathrm{b}}$ & $\varphi$ & $14.8^{\mathrm{c}}$ \\
& & $\tau$ & 30.5 \\
\hline
\end{tabular}

\begin{tabular}{lll}
\hline \multicolumn{2}{l}{ Rotational constants } \\
\hline & observed & calculated \\
\hline$A$ & 5198.2 & 5201.7 \\
$B$ & 4747.9 & 4744.4 \\
$C$ & 2710.9 & 2709.2
\end{tabular}

a Not a derived structure (see text).

b Assumed values. c Fitted to MW data.

atoms. The large difference between observed and calculated values can only be explained on the basis of a non-planar ring configuration. One further argument is the non-zero value of the dipole moment component in the near out-of-plane direction, $\mu_{c}=$ $0.278(8) \mathrm{D}$. This component would be zero if the ring atoms were coplanar.

While a complete structure cannot be obtained from the available data, the molecular conformation can be specified reliably because of the rotational constants alone are sufficient to eliminate conformations other than the twisted form (see Figure 2). Based on the limited experimental data, a number of assumptions are required in order to extract some of the gross structural features:

(1) Structural parameters were taken from related molecules such as cyclohexene [2] and 2,3-dihydrop-dioxin.

(2) The atoms $\mathrm{O}_{1}, \mathrm{C}_{2}, \mathrm{C}_{3}, \mathrm{C}_{4}$ and the hydrogens associated with the ethylene group are coplanar. 


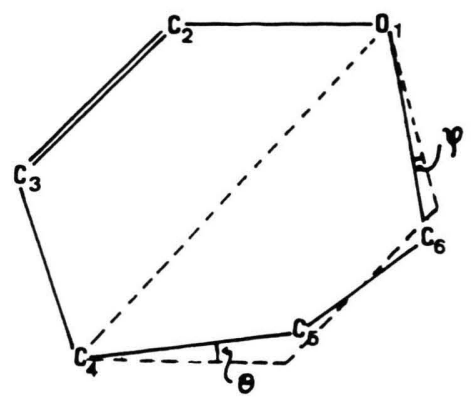

Fig. 2. The twisted conformation for the 3,4-dihydro-1,2pyran molecule giving the numeration of the ring atoms. Definition of the $\theta$ and $\varphi$ angles.

(3) All the $<\mathrm{HCH}$ angles associated with the methylene groups are equal and the $\mathrm{CH}_{2}$ plane bisects the $<\mathrm{CCC}$ and $<\mathrm{CCO}$ angles.

(4) All the $\mathrm{C}-\mathrm{H}$ bond distances are assumed to be equal and the hydrogens associated with the ethylene group lie in the bisector of $<\mathrm{C}_{4} \mathrm{C}_{3} \mathrm{C}_{2}$ and $<\mathrm{C}_{3} \mathrm{C}_{2} \mathrm{O}_{1}$.

With these assumptions the $\theta$ and $\varphi$ angles between the $\mathrm{C}_{4} \mathrm{C}_{5}$ and $\mathrm{O}_{1} \mathrm{C}_{6}$ bonds and their projections on the $\mathrm{O}_{1} \mathrm{C}_{2} \mathrm{C}_{3} \mathrm{C}_{4}$ plane (see Fig. 2) were fitted to the experimental rotational constants. The structural parameters and rotational constants are summarized in Table 4. These parameters, though not necessarily unique, reproduce the rotational constants quite well. The twisted angle $\tau$ between the $\mathrm{C}_{5} \mathrm{C}_{6}$ and $\mathrm{C}_{2} \mathrm{C}_{3}$ bonds for this structure is $30.5^{\circ}$.

Similar calculations were made with the bent form, but it was impossible to reproduce the experimental rotational constants with acceptable deviations.

\section{Vibrationally Excited States}

The vibrational satellites corresponding to the low frequency skeletal ring modes would be expected to be the most prominent ones in the excited states spectrum, according to the FIR and Raman spectra $(10,11)$. By far the most intense excited states are observed at frequencies close to those of the ground state transitions, and these form the basis of welldefined progressions, related both by essentially constant frequency increments and by steady diminution of intensity. From considerations of intensity and relationship discussed below, these families were assigned to successive quanta of the ringbending and ring-twisting vibrations, $v_{\mathrm{b}}$ and $v_{\mathrm{t}}$, respectively. The assignment was facilitated by the Stark effect pattern and by using the RFMWDR technique. The vibrational satellite pattern of the $3_{2,2} \leftarrow 22_{2,1}$ transition is shown in Figure 3. The lines are labeled by the vibrational state quantum numbers $\left(v_{\mathrm{t}}, v_{\mathrm{b}}\right)$, where $v_{\mathrm{t}}$ and $v_{\mathrm{b}}$ refer to the serial quantum numbers of the ring-twisting and ringbending vibrations, respectively.

Rotational constants and quartic centrifugal distortion constants for the first and second excited states of the bending mode and for the first excited state of the twisting mode, were obtained by using a model up to the first order centrifugal distortion as in the case of the ground state. Measured transitions of the remaining assigned excited states were analyzed by using the rigid rotor model. Rotational parameters are collected in Table 5. The assignment to out-of-plane ring modes is supported by a progressively more negative value of $\Delta_{v}$ as $v$ increases, a behaviour characteristic of such vibrations [20].

Table 5. Rotational constants $(\mathrm{MHz}), \Delta_{v}=I_{c}-I_{a}-I_{b}\left(\mathrm{amu} \mathrm{A}^{2}\right)$, centrifugal distortion constants $(\mathrm{kHz})$, standard deviation of the fits $(\mathrm{MHz})$, maximum value of $J$ and number of measured transitions of the vibrational satellites.

\begin{tabular}{|c|c|c|c|c|c|c|}
\hline & $(0,1)$ & $(0,2)$ & $(0,3)$ & $(1,0)$ & $(2,0)$ & $(1,1)$ \\
\hline$A$ & $5188.976(4)^{a}$ & $5180.128(7)$ & $5171.99(2)$ & $5192.758(4)$ & $5186.95(1)$ & $5191.26(1)$ \\
\hline$B$ & $4750.833(4)$ & $4753.550(7)$ & $4756.03(3)$ & $4743.592(4)$ & $4739.47(2)$ & $4746.19(3)$ \\
\hline C & $2711.164(4)$ & $2711.400(7)$ & $2711.84(5)$ & $2708.729(4)$ & 2706.42(4) & $2709.01(6)$ \\
\hline$\Delta_{v}$ & -17.3650 & -17.4869 & -17.6153 & -17.2889 & -17.3313 & -17.2784 \\
\hline$\Delta_{J}$ & $0.8(2)$ & $-2.9(3)$ & & $1.3(2)$ & & \\
\hline$\Delta_{J K}$ & $-2.144(3)$ & $-2.141(5)$ & & $-2.152(4)$ & & \\
\hline$\Delta_{K}$ & $1.10(2)$ & $1.11(3)$ & & $1.10(3)$ & & \\
\hline$\delta_{J}^{n}$ & $-0.0785(2)$ & $-0.0743(3)$ & & $-0.0829(4)$ & & \\
\hline$\delta_{K}$ & $1.165(4)$ & $1.169(5)$ & & $1.657(7)$ & & \\
\hline$\sigma$ & 0.023 & 0.031 & 0.210 & 0.024 & 0.166 & 0.176 \\
\hline$J_{\max }$ & 35 & 35 & 11 & 18 & 9 & 10 \\
\hline$N$ & 135 & 113 & 17 & 90 & 17 & 16 \\
\hline
\end{tabular}

\footnotetext{
a Standard errors in parenthesis on the last digit.
} 


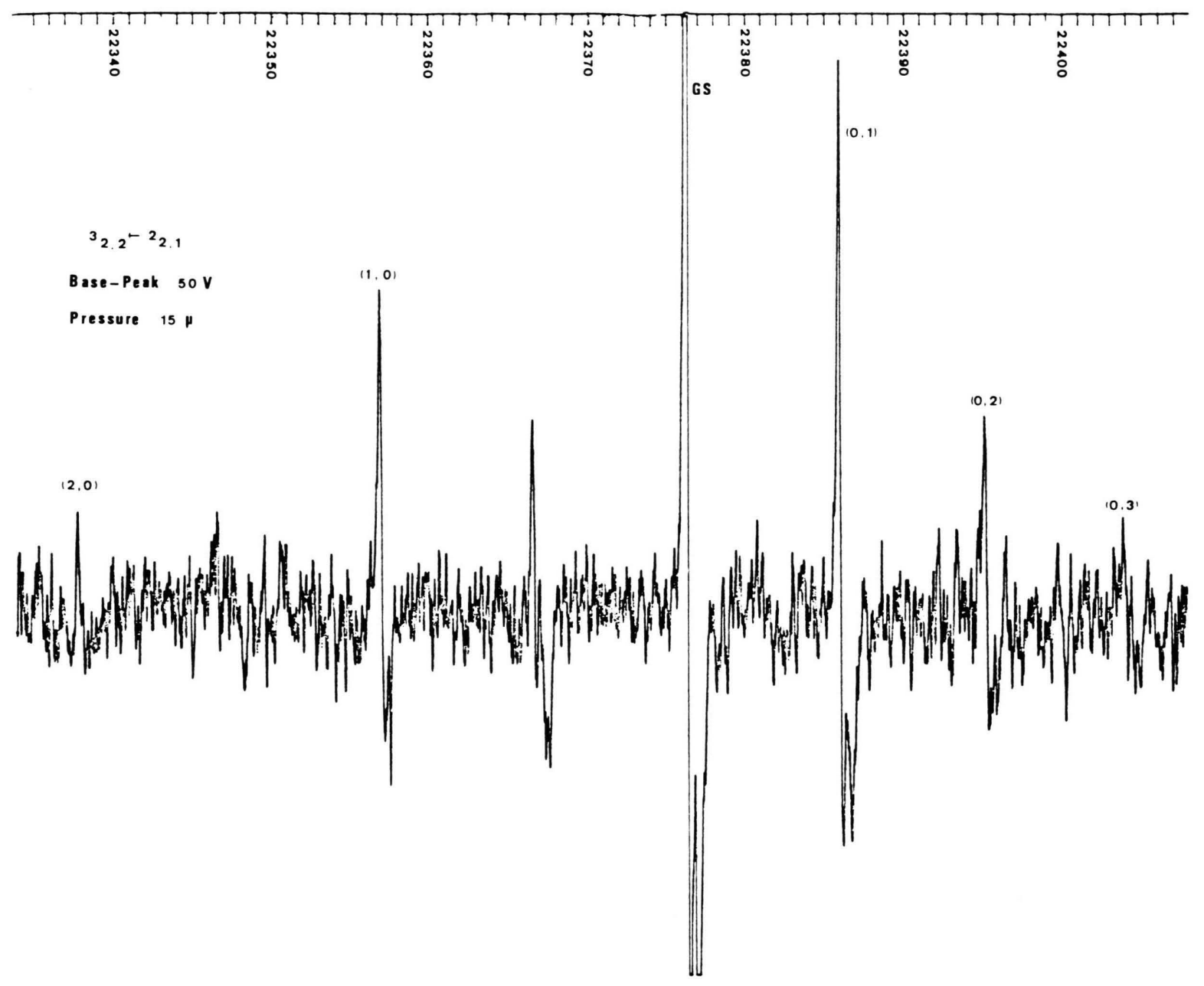

Fig. 3. Microwave absorption spectrum of 3,4-dihydro-1,2-pyran ( $3_{2,2} \leftarrow 2_{2,1}$ transition).

We undertook the determination of the energy and the nature of these vibrations from relative intensity measurements and their respective rotational constants. Because of the possibility of interference with other lines and their Stark components, relative intensity measurements [21], in a rich spectrum like this one, are difficult to make. The transitions initially selected were those for which the corresponding ones were isolated from other lines. This analysis was only possible for the ground state and for the $(0,1),(0,2)$ and $(1,0)$ excited states, because of the weakness of the satellites corresponding to other excited states. The average relative intensities obtained at room temperature are shown in Table 6. A comparison between the vibrational frequencies obtained from FIR [10] spectroscopy (see Table 6) and those obtained from microwave spectroscopy in this work shows that they are in an excellent agreement and confirms the microwave assignment.

Table 6. Relative intensities in 3,4-dihydro-1,2-pyran vibrational satellites.

\begin{tabular}{lllll}
\hline$\left(v_{\mathrm{t}}^{\prime}, v_{\mathrm{b}}^{\prime}\right) /\left(v_{\mathrm{t}}, v_{\mathrm{b}}\right)$ & $\begin{array}{l}\text { Num- } \\
\text { ber } \\
\text { of tran- } \\
\text { sitions }\end{array}$ & $\begin{array}{l}\text { Average rel. } \\
\text { intensity }\end{array}$ & $\begin{array}{l}\text { Vibra- } \\
\text { tional } \\
\text { separation } \\
\left(\mathrm{cm}^{-1}\right)\end{array}$ & $\begin{array}{l}\text { FIR fre- } \\
\text { quen- } \\
\text { cies } \\
\left(\mathrm{cm}^{-1}\right)^{\mathrm{a}}\end{array}$ \\
\hline$(0,1) /(0,0)$ & 22 & $0.42(0.03)^{\mathrm{b}}$ & $177(14)$ & 177.2 \\
$(0,2) /(0,1)$ & 16 & $0.43(0.04)$ & $172(18)$ & 175.8 \\
$(1,0) /(0,0)$ & 20 & $0.26(0.02)$ & $274(15)$ & 280.1 \\
\hline
\end{tabular}

a Taken from Ref. [10].

b Standard deviations in parentheses. 

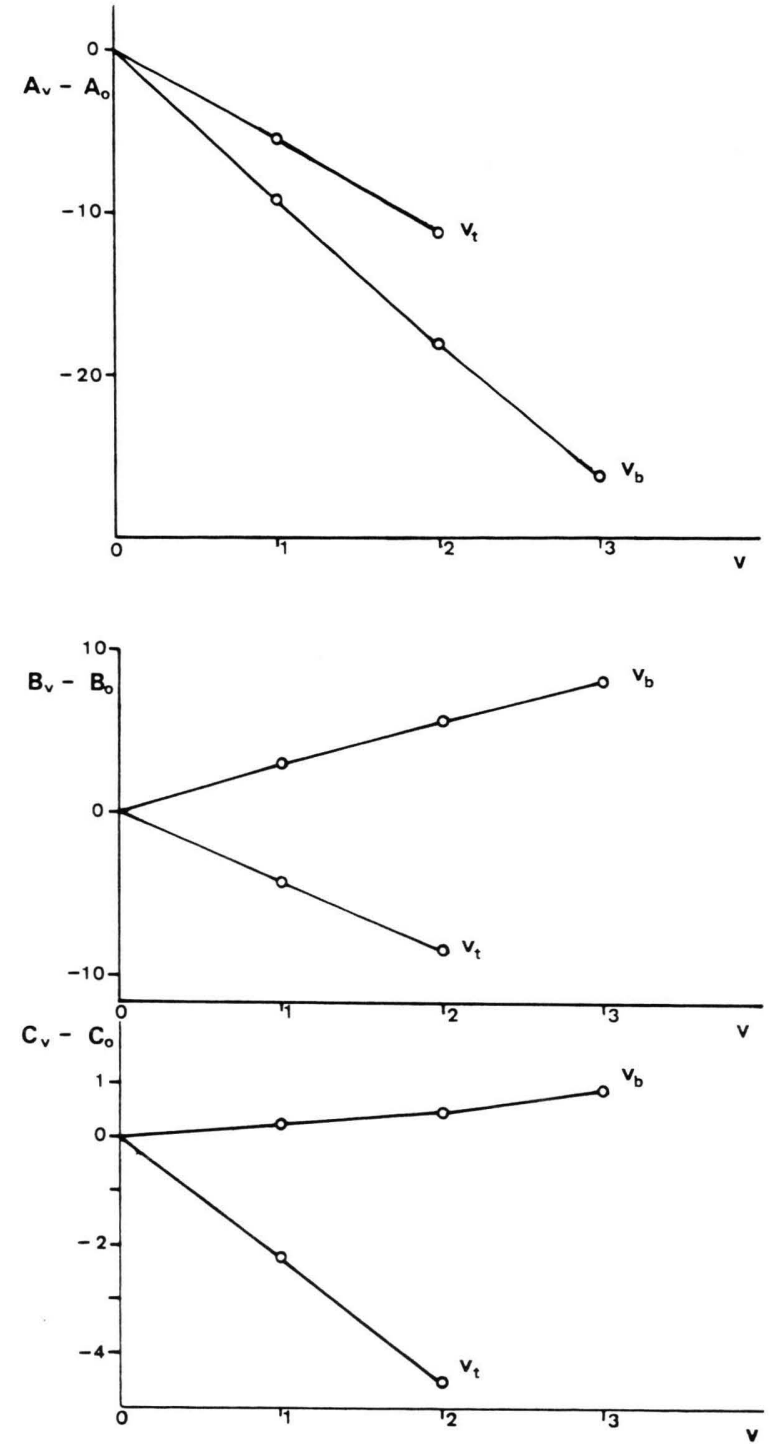

Fig. 4. Variation of rotational constants as a function of the ring-bending and ring-twisting vibrational quantum numbers. $v_{\mathrm{b}}$ and $v_{\mathrm{t}}$, respectively.

\section{Discussion}

The microwave spectrum of 3,4-dihydro-1,2pyran shows the twisted conformer to be the most stable one for this molecule. This agrees with the conclusions obtained from FIR [10] and Raman [11] spectrocopy. The estimated value of the twist angle, $\tau=30.5^{\circ}$, is quite similar to those determined in cyclohexene, $\tau=30.1^{\circ} \quad$ [2], 2,3-dihydro-p-dioxin. $\tau=29.9^{\circ}[3]$ and 3,6-dihydro-1,2-pyran, $\tau=31.5^{\circ}[4]$ from microwave spectroscopy. As in the case of 2,3 -dihydro-p-dioxin $[3,10]$, this microwave value is in marked disagreement with the FIR value of $51.04^{\circ}$ [10], obtained by computing the value of the twist angle corresponding to the minimum of the potential surface. The origin of this discrepancy between microwave and FIR values of this angle is attributed by Wells and Malloy [3] to the uncertainty in he FIR value associated with the difficulty of a precise knowledge of the dynamics of the vibration, which is very sensitive to the motion of the hydrogen atoms. A twisted form for 3,4-dihydro1,2-pyran is consistent with the reduction of the ring angle strain and torsional forces about single $\mathrm{C}-\mathrm{C}$ bonds that occur by reaching this conformation. A bent structure allows one to reduce some of the ring angle strain but does not achieve a staggered conformation of the $\mathrm{O}-\mathrm{CH}_{2}-\mathrm{CH}_{2}$ methylene groups.

The linear variation of the rotational constants in the successive bending and twisting excited states (see Fig. 4) and identification of the satellites without splitting in the excited states asigned prove that the barrier to interconversion of the most conformer is high. The convexity of these curves would indicate a slight anharmonicity of these modes. The same conclusions can be reached from the almost equal vibrational separations between the lower states of the bending mode. This is consistent with the height of the barriers to inversion determined from FIR $\left(2430 \mathrm{~cm}^{-1}\right)$ [10] and NMR $\left(2308 \mathrm{~cm}^{-1}\right)$ [13] spectroscopy, and with the FIR value [10] of the barrier to planarity $\left(6160 \mathrm{~cm}^{-1}\right)$.

Several unsuccesful searches have been done to find the spectrum attributable to the metastable bent form predicted to be present in about 18\% abundance at room temperature from FIR spectroscopy [10]. This value is an extrapolation of the potential surface as determined from eigenvalues near the potential minimum, and consequently its uncertainty is much greater than that of the barrier heights determined in the same way. This uncertainty affects therefore the intensity of the lines corresponding to this predicted form. On the other hand, the sensitivity of the rotational spectrum to the assumed structural parameters and the high density of the observed spectrum renders these searches very difficult. However, we cannot rule out the presence of this metastable bent form on the basis of our results. 
J. C. López and J. L. Alonso - The Microwave Spectrum of 3,4-Dihydro-1,2-Pyran

[1] T. Ogata and K. Kozima, Bull. Chem. Soc. Japan 42, 1263 (1969).

[2] L. H. Scharpen, J. E. Wollrab, and D. P. Ames, J. Chem. Phys. 49, 2368 (1968).

[3] J. A. Wells and T. B. Malloy, Jr., J. Chem. Phys. 60, 2132 (1974).

[4] J. A. Wells and T. B. Malloy, Jr., J. Chem. Phys. 60, 3987 (1974).

[5] J. Boëseken and W. J. F. de Rijck van der Gracht, Rec. Trav. Chim. 56, 1203 (1937).

[6] C. V. Berket, N. V. Freeman, and K. S. Pitzer, J. Amer. Chem. Soc. 70, 4227 (1948).

[7] C. Chen, R. LeFevre, and K. Sundaran, J. Amer. Chem. Soc. 87, 553 (1965).

[8] F. A. L. Anet and M. Z. Hag, J. Amer. Chem. Soc. 87, 3147 (1965).

[9] N. Neto, C. Dilanzo, E. Castelluci, and S. Califano, Spectrochim. Acta A 23, 1763 (1967).

[10] R. C. Lord, T. C. Rounds, and T. Ueda; J. Chem. Phys. 57, 2572 (1972).

[11] J. R. Durig, R. O. Carter, and L. A. Carreira, J. Chem. Phys. 60, 3098 (1974).
[12] T. Ikeda and R. C. Lord, J. Chem. Phys. 56, 4450 (1972).

[13] C. H. Bushweller and J. W. O'Neil, Tetrahedrom Lett. 1969, 4713.

[14] J. Sheridan, in "Physical Methods in Heterocyclic Chemistry" (A. R. Katritzky, Ed.), Vol. VI, Chapter 2, Academic Press, New York 1974.

[15] F. J. Wodarczyk and E. B. Wilson, Jr., J. Mol. Spectrosc. 37, 445 (1971).

[16] J. K. G. Watson, J. Chem. Phys. 46, 1935 (1967).

[17] G. W. King, R. M. Hainer, and P. C. Cross, J. Chem. Phys. 11, 27 (1943).

[18] S. Golden and E. B. Wilson, Jr., J. Chem. Phys. 16, 669 (1948).

[19] J. S. Muenter, J. Chem. Phys. 48, 4544 (1968).

[20] K. Kuchitsu, T. Oka, and Y. Morino, J. Mol. Spectrosc. 15, 51 (1965).

[21] A. S. Esbitt and E. B. Wilson, Jr., Rev. Sci. Instrum. 34, 901 (1963). 